\title{
Peritoneal Dialysis in the Elderly: Modality of Choice During COVID-19 Pandemic
}

\author{
N Ozlem Harmankaya ${ }^{1 *}$ and Hakan Kaptanogullari ${ }^{2}$ \\ ${ }^{1}$ Department of Internal Medicine, Division Nephrology, Biruni University Medical Faculty, Turkey \\ ${ }^{2}$ School of Dialysis Technicians, Vocational School of Health Sciences, Biruni University, Turkey
}

*Corresponding author: N Ozlem Harmankaya, Biruni University Medical Faculty, Istanbul, Turkey.

To Cite This Article: Harmankaya NO, Kaptanogullari H. Peritoneal Dialysis in the Elderly: Modality of Choice During COVID-19 Pandemic. 2020 9(3). AJBSR.MS.ID.001397. DOI: 10.34297/AJBSR.2020.09.001397.

Received: 㘹: May 28, 2020; Published: 眥 July 01, 2020

\section{Introduction}

At the time of the COVID-19 pandemic, the elderly is one of the vulnerable patient groups for whom treatment at home would be most beneficial. Adding to this the fact that elderly people are under stay-at-home orders in a considerable number of countries as of now, the need for home dialysis becomes even more pronounced. The number of older patients with end-stage kidney disease undergoing renal replacement therapy is increasing worldwide. Elderly patients with additional comorbidities are now surviving long enough to be started on dialysis [1]. According to most endstage renal disease (ESRD) data registries, elderly patients account for about $25 \%$ to $30 \%$ of dialysis patients around the globe [2]. In the United States, $49 \%$ of patients initiating renal replacement therapy are 65 years of age or older [3]. In Canada, Europe, and Australia/ New Zealand, a significant percentage of patients initiating dialysis are 65 years of age or older $(84 \%, 56 \%$, and $46 \%$, respectively [4].

For the majority of elderly patients with renal failure, the choice of therapy has been in-center dialysis with 96\% in-center hemodialysis, $3.5 \%$ continuous ambulatory peritoneal dialysis/ continuous cyclic peritoneal dialysis and only $0.3 \%$ home hemodialysis [5]. In 2017, 62.7\% of all prevalent ESRD patients were receiving hemodialysis therapy, $7.1 \%$ were being treated with peritoneal dialysis. Among patients being treated with hemodialysis, 98.0\% used in-center hemodialysis, and 2.0\% used home hemodialysis [3].

To facilitate treatment at home for the elderly, peritoneal dialysis is suggested as the modality of choice. The implementation of peritoneal dialysis modality for the elderly, where possible, must be encouraged. Peritoneal dialysis is a comparable modality to manage elderly ESRD patients, considering the psychological and its comparable outcomes to hemodialysis. A major advantage of peritoneal dialysis is that there is no need to establish vascular access with invasive vascular procedures. In addition, peritoneal dialysis has better preservation of residual renal function compared to hemodialysis. Peritoneal dialysis provides better blood pressure control and improved middle molecular clearance. The removal of middle molecules allows elderly patients to have a more liberal diet, reducing the risk of malnutrition by imposing strict protein and other dietary restrictions.

Transporting frail elderly patients to hemodialysis centers three times per week poses challenges, especially ensuring COVID-19 prevention measures are taken. Home dialysis is the modality of choice for the added benefit of social isolation during the COVID-19 pandemic. The advantages of peritoneal dialysis include that it requires less visits to medical centers/hospitals, can be performed by the patient or a caregiver and that it does not require invasive vascular procedures.

Older people experience various problems related to deterioration of physical function. They also experience deterioration of psychological or psychiatric conditions. Comorbidities, disabilities, lack of dexterity, and lack of assistance are factors which may limit the use of peritoneal dialysis in the elderly. Assisted peritoneal dialysis performed by family members or caregivers offers a convenient solution to these problems faced by elderly patients. Telecommunication technologies (Telemedicine) can be utilized to supervise peritoneal dialysis procedures.

The principal advantage of peritoneal dialysis is the ability to provide treatment at home and thereby avoid the disruption and discomfort of visits to hospital and dialysis centers which may 
cause a danger to the health of elderly patients. Quality of life and staying safe at home are the two main goals of home dialysis modalities during the COVID-19 pandemic. Fewer hospital visits, less exposure to other patients/people are advantages of peritoneal dialysis for elderly patients who are much more susceptible to infections. Peritoneal dialysis has the potential to meet specific needs of the elderly and provide a safer alternative to hemodialysis during COVID-19.

\section{Reference}

1. Auguste BL, Chan CT (2019) Home Dialysis Among Elderly Patients: Outcomes and Future Directions. Canadian Journal of Kidney Health and Disease 6: 1-6.
2. Canaud B, Tong L, Tentori F, Takashi Akiba, Angelo Karaboyas, et al. (2011) Clinical practices and outcomes in elderly hemodialysis patients: results from the Dialysis Outcomes and Practice Patterns Study (DOPPS). Clin J Am Soc Nephrol 6(7): 1651-1662.

3. United States Renal Data System (2017) Usrds Annual Data Report: Epidemiology of Kidney Disease in the United States. National Institutes of Health, National Institute of Diabetes and Digestive and Kidney Diseases; Bethesda, MD, USA.

4. Ahmed FA, Catic AG (2018) Decision-Making in Geriatric Patients with End-Stage Renal Disease: Thinking Beyond Nephrology. J Clin Med 8(1):

5. Ross DL, Schrag WF, Pond P (2016) The Pros and Cons of Home vs Incenter Dialysis in the Elderly. In Dialysis in Older Adults, ed. Madhukar Misra p. 29-44. 